\title{
Analytic proof of the Weinberg theorem for off mass shell and composite pions
}

\author{
Poster for the 5th "Quark confinement and the hadron spectrum" Conference, Gargnano, September 2002 \\ Pedro Bicudo \\ Departamento de Física, and CFIF, Instituto Superior Técnico, Av. Rovisco Pais, 1049-001 Lisboa, Portugal
}

\begin{abstract}
The Weinberg theorem for the pion-pion scattering is proved analytically in full detail for quark models in the ladder truncation. The proof is displayed with Feynman diagrams. The axial and vector Ward identities, for the quark propagator and for the ladder, exactly cancel any model dependence. Off mass shell and finite size effects are included in the quark-antiquark pion Bethe Salpeter vertices. This is applied to compute the isospin 0, 1 and 2 scattering matrices off the mass shell .
\end{abstract}

The success of the Quark Model [1] relies on its ability to reproduce the Hadron spectrum with microscopical interacting quarks. This includes for instance the linear Regge trajectories, and the correct spin-spin and spinorbit interactions. Moreover the quark model is competent to predict microscopically the hadron-hadron interactions [2]. For recent coupled channel studies see [3, 4, 5]. However the quark model suffered from initial difficulties of fully understanding the low pion mass and the small pi-pi scattering length. With the aim to cure this important problem, chiral symmetry breaking was introduced in the quark model. [6, 7, 8]. This opened the possibility to extend the low energy pion theorems of current algebra to the quark model framework.

In this paper we prove that the $\pi-\pi$ scattering theorem of Weinberg [9] applies to quark models with chiral invariant quark-quark interactions, including the Nambu and Jona-Lasinio model [10,11], conventional quark models with confining instantaneous interactions [4, [], models with Euclidean space integrations [12,5, 13, , and also covariant models in Minkowsky space [14]. After the original work of Weinberg [9], the theorem was also derived with Ward identities for the pion fields [15] and with a functional integration of quarks 16 . However these methods use effective pions, whereas pions in quark models have naturally a finite size 17] which might affect $\pi-\pi$ scattering.

Here we complete in full detail an analytical proof which was recently outlined in [5], using Ward identities and the diagrammatic method [18]. Moreover we extend the proof to pions with off mass shell momenta. Amplitudes for off mass shell pions are crucial because $\pi-\pi$ scattering is experimentally estimated from $\pi-N$ scattering. The nucleon $N$ provides a virtual off mass shell pion $\pi^{*}$ that scatters with the incident $\pi$. For instance a virtual pion $\pi^{*}$ with offshellness $P^{2}-M_{\pi}^{2}=-3.32 M_{\pi}^{2}$ is present in the $\pi$ production at threshold.

To prove the Weinberg theorem in the Quark Model formalism we have to truncate the series of Feynman di- agrams consistently. The ladder truncation is commonly assumed for the boundstate equations,

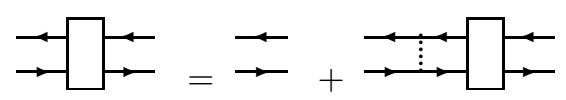

where the dotted line corresponds to the chiral invariant quark-quark interaction of vertex $V$ and kernel $\mathcal{K}$. The arrowed line corresponds to the Dirac quark propagator. The Ward identities 19] show that the ladder is consistent with the quark self energy equation in the rainbow approximation. When chiral symmetry is spontaneously broken, the dressed quark propagator is non trivial,

$$
S=\frac{i}{A\left(k^{2}\right) \not k-B\left(k^{2}\right)},
$$

and the $\pi$ is a Goldstone boson in the chiral limit. There is also evidence that the hadron-hadron coupled channel equations should include one meson exchange, both in the sigma model [9,20], in the Nambu and Jona-Lasinio model [11], in the constituent quark models [5], and in an Euclidean quark model [5]. In microscopic calculations the Feynman diagram for $\pi-\pi$ scattering must therefore include inside the box a vertical scalar ladder and a horizontal scalar ladder, see paper [5] for details. To cure double counting we also have to subtract an empty box.

The main task of this paper consists in computing independently of the Quark Model, and up to order $M_{\pi}^{2}$ and $P_{i} P_{j}$ in the $\pi$ mass and momenta, the Feynman loop,

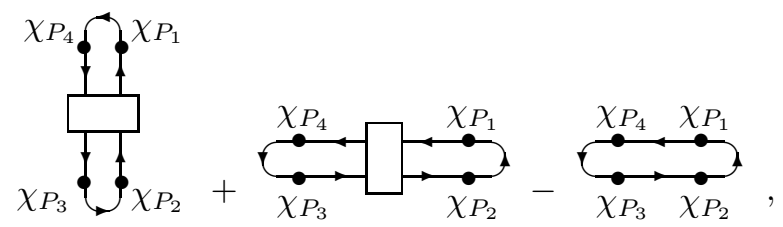

where $\chi$ is the Bethe Salpeter vertex of the pion. The subindex $P i$ accounts an external momentum flowing into the loop.

To derive the proof it is convenient to define the dressed axial vertex $\Gamma_{A}$ with the axial Ward identity,

$$
\Gamma_{A}\left(k_{1}, k_{2}\right)=S^{-1}\left(k_{1}\right) \gamma_{5}+\gamma_{5} S^{-1}\left(k_{2}\right)
$$

because it is related to the pion vertex and because it has the crucial property,

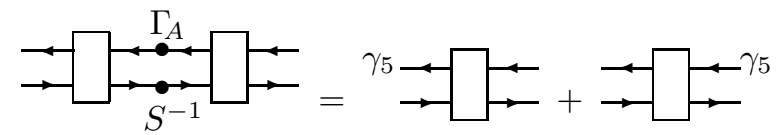

which constitutes a Ward identity for the ladder. This identity is derived if we expand the ladders and substitute 
the vertex in the left hand side. Then all terms with an intermediate $\gamma_{5}$ include the anticommutator $\left\{\gamma_{5}, V\right\}$ and this cancels because the interaction is chiral invariant and the kernel is local. Only the right hand side survives.

We now derive a second useful relation for $\Gamma_{A}$. The Dyson Schwinger equation for the full propagator is,

$$
S(k)^{-1}=S_{0}^{-1}(k)-\int \frac{d^{4} q}{(2 \pi)^{4}} \mathcal{K}(q) V S(k+q) V
$$

where $V$ is some vertex that anticommutes with $\gamma_{5}$ and that is color dependent as well, $\mathcal{K}$ is the quark-quark Kernel, and $S_{0}=i /(\not k+m)$ is the bare quark propagator. If we multiply right or left with $\gamma_{5}$ and sum, we get,

$$
\begin{gathered}
S\left(k_{1}\right)^{-1} \gamma_{5}+\gamma_{5} S\left(k_{2}\right)^{-1}=S_{0}\left(k_{1}\right)^{-1} \gamma_{5}+\gamma_{5} S_{0}\left(k_{2}\right)^{-1} \\
\quad+\int \mathcal{K}(q) V\left(S\left(k_{1}+q\right) \gamma_{5}+\gamma_{5} S\left(k_{2}+q\right)\right) V
\end{gathered}
$$

and this is the Bethe Salpeter equation for the vertex,

$$
\begin{aligned}
& \Gamma_{A}\left(k_{1}, k_{2}\right)=\gamma_{A}\left(k_{1}, k_{2}\right)+\int \mathcal{K}(q) V S\left(k_{1}+q\right) \Gamma_{A} S\left(k_{2}+q\right) V \\
& \gamma_{A}\left(k_{1}, k_{2}\right)=S_{0}^{-1}\left(k_{1}\right) \gamma_{5}+\gamma_{5} S_{0}^{-1}\left(k_{2}\right),
\end{aligned}
$$

where the particular part $\gamma_{A}$ is the bare axial vertex,

$$
\gamma_{A}=\frac{(P-2 m)}{i} \gamma_{5}, \quad P=k_{1}-k_{2} .
$$

$\gamma_{A}$ vanishes when the current quark mass $m$ is small (chiral limit) and at the same time the total momentum $P^{\mu}$ of the vertex is small. On the other hand the dressed vertex $\Gamma_{A}$ is finite,

$\Gamma_{A}\left(k_{1}, k_{2}\right)=\frac{A\left(k_{1}\right) k_{1}-A\left(k_{2}\right) k_{2}-B\left(k_{1}\right)-B\left(k_{2}\right)}{i} \gamma_{5}$,

providing spontaneous chiral symmetry breaking occurs in eq. (6) to generate a dynamical mass in the dressed quark propagator. After we iterate the Bethe Salpeter equation for the dressed axial vertex $\Gamma_{A}$, and include the external propagators, we get the second useful relation,

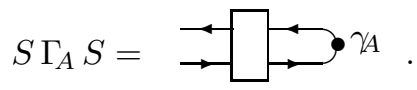

The main step to get the proof consists in decreasing the number of vertices with the help of eqs. (5) and (11),

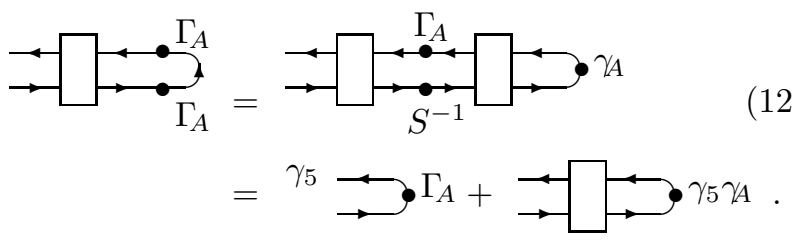

This is repeated to compute the square box with ladder,

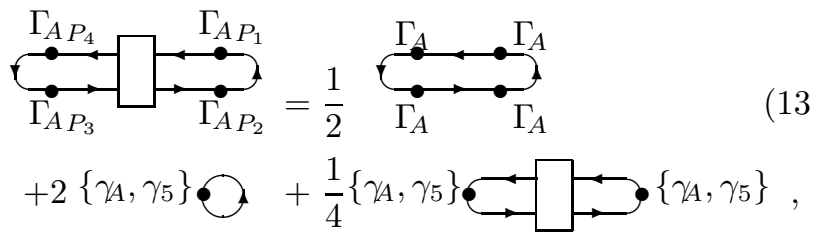

and we get three terms, respectively of order $1, \gamma_{A}$ and $\gamma_{A}^{2}$. We note that all the other factors $\left(\Gamma_{A}, S\right.$, and the scalar and vector ladder) are finite and carry the scale of the effective quark-quark interaction.

When we sum the three diagrams of the full box,

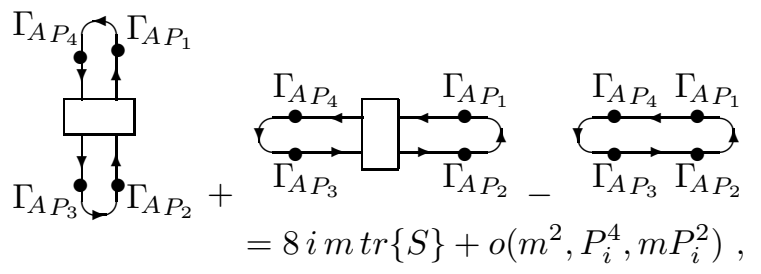

the finite term of order 1 exactly cancels. The total box vanishes in the chiral limit of $m=0$, although each one of the three boxes is generally finite and proportional to the fourth power of the scale of the interaction. This cancellation in eq. (15) complies with the Adler zero and corresponds to a higher Ward identity.

The dependence of eq. (15) on the quark current mass $m$ is trivially computed with eqs. (9) and (13). The exact cancellation in the momentum dependence is more subtle. Expanding up the first order in $P_{i} P_{j}$, the $\gamma_{A}$ and $\gamma_{A}^{2}$ terms in eq. (13),

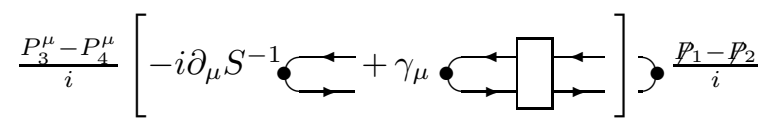

and this cancels due to the vector Ward Identity which has and analogous variant to eq. (11).

We now apply these Ward identity techniques to the actual Feynman amplitude with pion vertices of eq. (3). In the limit of vanishing current quark mass $m$ and vertex momentum $P^{\mu}$, the Bethe-Salpeter (8) equation for the axial vertex $\Gamma_{A}$ becomes homegenous and is thus identical to the homogeneous Bethe-Salpeter equation for the pion vertex $\chi_{\pi P}(k)$. In this limit the pion is a massless Goldstone boson, and the pion Bethe Salpeter vertex is proportional to the dressed axial vertex, and to the dynamical quark mass $B(k)$,

$$
\chi_{0}(k)=\frac{B(k)}{n_{\pi}} \gamma_{5}=\frac{1}{2 i n_{\pi}} \Gamma_{A 0}(k, k),
$$

where $n_{\pi}$ is the norm. And the loop (3) vanishes.

To get the loop (3) up to order $P_{i}^{2}$ and $M_{\pi}^{2}$, we need at most two full Bethe Salpeter vertices $\chi$, the other two can be approximated by $\Gamma_{A} /\left(2 i n_{\pi}\right)$. Expanding up to second order in $\chi-\Gamma_{A} /\left(2 i n_{\pi}\right)$ and regrouping the sum, we find that the amplitude of eq.(33) is the sum of four classes of terms. Each class includes a sum of the possible cyclic permutations of the external momenta $P_{1}, P_{2}, P_{3}$, and $P_{4}$. We get, eq.( 14$)$ with factor 3 times $\left(2 i n_{\pi}\right)^{-4}$, minus, with factor 2 times $\left(2 i n_{\pi}\right)^{-3}$, (4 permutations)

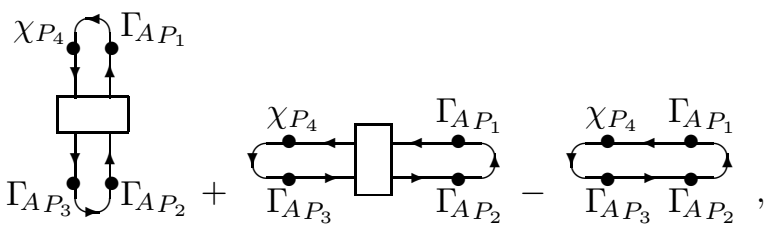


plus, with factor $\left(2 i n_{\pi}\right)^{-2},(4$ permutations $)$

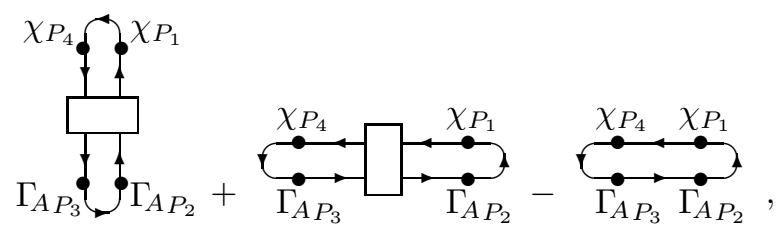

plus, with factor $\left(2 i n_{\pi}\right)^{-2},(2$ permutations $)$

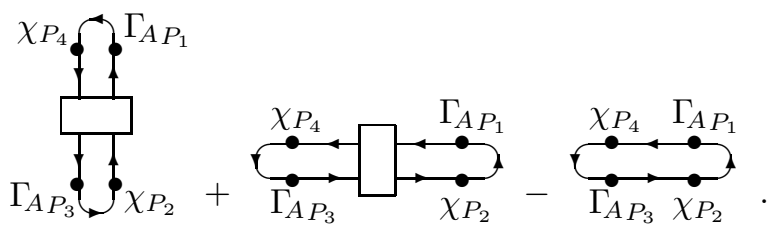

To repeat the main step of eq. (12), replacing one of the two $\Gamma_{A P}$ by a $\chi_{P}$, it is convenient to include (or remove) a ladder in the pion vertex $\chi_{P}$. In the neighborhood of a boundstate $b$ pole $M_{b}^{2}$ in the external momentum $P^{2}$,

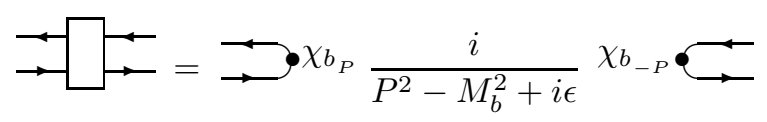

multiplying the right of both sides with the vertex $\chi$,

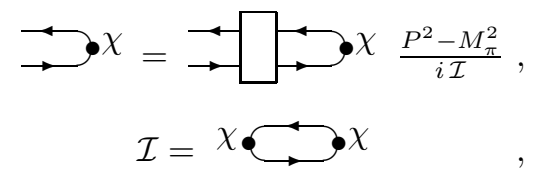

where $\mathcal{I}$ is finite and proportional to the square of the scale of the interaction, nevertheless it will factorize from the results. Moreover the off mass shell Bethe Salpeter equation is derived replacing eq. (22) in eq. (11),

$$
\varkappa \chi_{P}=\chi_{P}\left(1-\frac{P^{2}-M_{\pi}^{2}}{i \mathcal{I}}\right)^{-1} \text {. }
$$

The off mass shell correction only contributes to the Bethe Salpeter equation (23) at the order $P^{2}$ and $M^{2}$. Therefore, up to the first order in the external $P^{\mu}$, the vertex $\chi_{P}$ is formally the same function of $P^{\mu}$, both for mass shell and for off mass shell pions.

The diagrams eventually simplify into traces related to the pion decay relation 21,

$$
\operatorname{tr}\left\{(S \chi S)_{P} \gamma^{\mu} \gamma^{5}\right\}=2 f_{\pi} P^{\mu}
$$

which measures $f_{\pi}$ in the electroweak decay of pions on the mass shell. Because off mass shell vertices $\chi_{P}$ are formally the same as the mass shell ones, eq. (24) is also correct for $P^{2} \neq M^{2}$. This is experimentally confirmed with the Goldberger Treiman relation [22]. Another useful trace is directly extracted, substituting the spectral decomposition of the ladder (21) in eq. (11),

$$
\operatorname{tr}\left\{(S \chi S)_{-P} \gamma_{A_{P}}\right\}=2 n_{\pi}\left(P^{2}-M_{\pi}^{2}\right) .
$$

A detailed momentum analysis of eqs (24,25) shows that the norm $n_{\pi}=i f_{\pi}$ and produces the desired traces,

$$
\begin{aligned}
\operatorname{tr}\left\{(S \chi S)_{P_{1}} \gamma_{A P_{2}}\right\} & =-2 n_{\pi}\left(P_{1} \cdot P_{2}+M_{\pi}^{2}\right), \\
-\operatorname{mtr}\{S\} & =n_{\pi}^{2} M_{\pi}^{2},
\end{aligned}
$$

including the Gell-Mann Oakes and Renner relation [23.

This completes the necessary steps to compute the loop (3). The diagrams of eq. (14) are trivial to compute, completing eq. (15) with the Gell-Mann Oakes and Renner relation (27). The three other cases (18, 19,20) are now straightforwardly computed with the techniques that lead to eq. (13), replacing eq. (11) by eq. (22). It is clear that when we replace a $\gamma_{A}$ by $\chi\left(P_{j}^{2}-M_{\pi}^{2}\right) / i I$, the order in $P_{j}$ increases. Therefore the trace that replaces the term with a pair of $\left\{\gamma_{A}, \gamma_{5}\right\}$ in eq. (13) now vanishes up to the order of $P_{i} P_{j}$ and of $M_{\pi}^{2}$. The trace that replaces the term of order $\left\{\gamma_{A}, \gamma_{5}\right\}$ in eq. (13) is now computed with the trace (26). Summing the total contributions to the Feynman loop of eq. (3) we get,

$$
\begin{gathered}
+3\left(\frac{1}{2 i n_{\pi}}\right)^{4}\left(-8 i n_{\pi}^{2} M_{\pi}^{2}\right) \\
-2\left(\frac{1}{2 i n_{\pi}}\right)^{3} \sum_{4 \text { perm. }} 2 n_{\pi}\left(P_{1}^{2}+P_{1} \cdot P_{3}-2 M_{\pi}^{2}\right) \\
+\left(\frac{1}{2 i n_{\pi}}\right)^{2} \sum_{4 \text { perm. }} \frac{P_{1}^{2}+P_{2}^{2}+P_{1} \cdot P_{2}+P_{1} \cdot P_{3}+P_{2} \cdot P_{4}-2 M_{\pi}^{2}}{i} \\
+\left(\frac{1}{2 i n_{\pi}}\right)^{2} \sum_{2 \text { perm. }} \frac{P_{1}^{2}+P_{3}^{2}-2 M_{\pi}^{2}}{i} \\
=\frac{i}{4 f_{\pi}^{2}}\left[\left(P_{1}+P_{2}\right)^{2}+\left(P_{1}+P_{4}\right)^{2}-2 M_{\pi}^{2}\right],
\end{gathered}
$$

where the conservation $P_{1}+P_{2}+P_{3}+P_{4}=0$ of momentum was used to simplify the result.

We finally compute the $\pi-\pi$ scattering matrix $\mathrm{T}$. We simply have to match the external pions, $i 1$ and $i 2$ incoming and $o 1$ and $o 2$ outgoing, with the four pion vertex that we just computed in eq.(28),

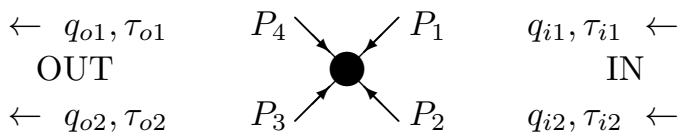

where the loop (3) is represented by the full circle. The loop is topologically invariant for cyclic permutations of $P_{1}, P_{2}, P_{3}$ and $P_{4}$. To remove double counting we fix one match, say $P_{1}=q_{i 1}$. Then there are six different combinations of the remaining external legs.

In what concerns color, all the combinations are identical because the pion is a color singlet, and the color factor is appropriately included in the definition of $f_{\pi}$, see eq. (24). In what concerns momentum, we express our result in the usual Mandelstam relativistic invariant variables $s, t$ and $u$. For instance the direct combination in eq. (29) produces the result $i\left(s+u-2 M_{\pi}^{2}\right) /\left(4 f_{\pi}^{2}\right)$. We now introduce flavor. For simplicity it was not regarded in the previous definitions of the vertices $\Gamma_{A P}$ and $\chi_{P}$. Because the pion is an isovector, we have three different cases $I=0, I=1$ and $I=2$. The flavor contributions 
to the pion vertex simply factorize from the momentum contribution, and the different combinations only produce two classes of flavor traces, see Table il. Summing the six possible combinations of color, spin, momentum and flavor traces, and dividing by $-i$, we finally get the $\pi-\pi$ scattering $T^{I}$ matrix,

$$
\begin{aligned}
& T^{0}=-\frac{2 s-M_{\pi}^{2}}{2 f_{\pi}^{2}}-\frac{s+t+u-4 M_{\pi}^{2}}{2 f_{\pi}^{2}}, \\
& T^{1}=-\frac{t-u}{2 f_{\pi}^{2}}, \\
& T^{2}=-\frac{-s+2 M_{\pi}^{2}}{2 f_{\pi}^{2}}-\frac{s+t+u-4 M_{\pi}^{2}}{2 f_{\pi}^{2}},
\end{aligned}
$$

where $s+t+u-4 M_{\pi}^{2}$ expresses the off mass shell contribution. We remark that eq. (30) complies with the Gasser and Leutwyler results. 15]. The $T^{I}$ matrices of eq. (30) are computed at the tree level (including scalar and vector $\mathrm{s}, \mathrm{t}$, and $\mathrm{u}$ exchange), which is exact up to the order of $P_{i}^{2} P_{j}^{2}$ and of $M_{\pi}^{2}$.

The $\pi-\pi$ mass shell scattering lengths $a_{0}^{I}$ are simply obtained from the scattering amplitudes $T^{0}, T^{2}$ with the Born factor of $\frac{-1}{16 \pi M_{\pi}}$, and for vanishing 3 -momenta. The $I=1$ case is antisymmetric so the first scattering parameter is $a_{1}^{1}$ and the corresponding factor is $\frac{-1}{16 \pi M_{\pi}} \frac{4}{3(t-u)}$,

$$
\begin{aligned}
& a_{0}^{0}=\frac{7}{32 \pi} \frac{M_{\pi}}{f_{\pi}^{2}}, \\
& a_{1}^{1}=\frac{1}{24 \pi} \frac{1}{M_{\pi} f_{\pi}^{2}}, \\
& a_{0}^{2}=\frac{-1}{16 \pi} \frac{M_{\pi}}{f_{\pi}^{2}},
\end{aligned}
$$

this is the result of the famous Weinberg theorem for $\pi-\pi$ scattering [9]. Off mass shell effects are very important for the experiments. For instance in the scattering at $\pi-\pi$ threshold of a $\pi$ beam with virtual $\pi^{*}$ provided by a nucleon target, we estimate that the off mass shell effects of eq. (30) decrease $a_{0}^{0}$ by a factor of 0.5 and increase $a_{0}^{2}$ by a factor of 1.7 .

We conclude that the Weinberg theorem for pionpion low energy scattering is also exact when the pions are off the mass shell and have a finite size. It is remarkable that the current quark mass $m$ and the traces $\operatorname{tr}\{S\}, \operatorname{tr}\left\{\left(S \Gamma_{A}\right)^{4}\right\}, \operatorname{tr}\left\{(S \chi)^{2}\right\}$, which depend on the finite scale of the interaction, say the string tension $\sigma$ or $\Lambda_{Q C D}$, disappear from the final result. Any quark model with a chirally symmetric interaction complies with the Weinberg theorem. This detailed proof confirms that Goldstone bosons non only are massless but also are noninteracting at low energy.

I acknowledge Emilio Ribeiro for reporting on nontrivial Ward identities. I also acknowledge that discussions with the collaborators of the recent works [3, 4, 5 , motivated the search for an analytical proof of the Weinberg theorem and identified the class of diagrams that should be included in the box of eq. (3).
[1] A. De Rujula, H. Georgi and S. L. Glashow, Phys. Rev. D 12, 147 (1975).

[2] J. Ribeiro, Z. Phys. C 5, 27 (1980).

[3] P.Bicudo, G. Krein and J. Ribeiro, Phys. Rev. C 64, 025202 (2001) arXiv:hep-ph/0105289;

[4] P.Bicudo, M. Faria, G. Marques, and J. Ribeiro arXiv:nucl-th/0106071.

[5] P. Bicudo, S. Cotanch, F. Llanes-Estrada, P. Maris, E. Ribeiro and A. Szczepaniak, Phys. Rev. D 65, 076008 (2002) arXiv:hep-ph/0112015.

[6] A. Le Yaouanc, L. Oliver, O. Pene and J.-C. Raynal, Phys. Rev. D 29, 1233 (1984); Phys. Rev. D 31, 137 (1985).

[7] S. Adler and A. Davis, Nucl. Phys. B 244, 469 (1984).

[8] P. Bicudo and J. Ribeiro, Phys. Rev. D 42, 1611 (1990); Phys. Rev. D 42, 1625 (1990); Phys. Rev. D 42, 1635 (1990).

[9] S. Weinberg, Phys. Rev. Lett. 17, 616 (1966).

[10] Y. Nambu and J. Jona-Lasinio, Phys. Rev. 124, 246 (1961); Phys. Rev. 122, 345 (1961).

[11] V. Bernard, U. G. Meissner, A. Blin and B. Hiller, Phys. Lett. B253, 443 (1991); V. Bernard, A. H. Blin, B. Hiller, Y. P. Ivanov, A. A. Osipov and U. Meissner, Annals Phys. 249, 499 (1996) arXiv:hep-ph/9506309.

[12] Y. Dai, C. Huang and D. Liu, Phys. Rev. D 43, 1717 (1991).

[13] S. Cotanch and P.Maris, arXiv:hep-ph/0210151.

[14] V. Sauli, arXiv:hep-ph/0108160.

[15] J. Gasser and H. Leutwyler, Annals Phys. 158, 142 (1984).

[16] C. Roberts, R. Cahill, M. Sevior and N. Iannella, Phys. Rev. D 49, 125 (1994) arXiv:hep-ph/9304315.

[17] S. R. Amendolia et al., Phys. Lett. B 146, 116 (1984), E. B. Dally et al., Phys. Rev. Lett. 48, 375 (1982).

[18] P. Bicudo, Phys. Rev. C 60, 035209 (1999) arXiv:nuclth/9802058.

[19] P. Bicudo, D.-S. Liu, J. Ribeiro, J. Villate Phys. Rev. D 47, 1145 (1993).

[20] V. De Alfaro, S. Fubini, G. Furlan, C. Rosseti, "Currents in Hadron Physics" , Amsterdam, North-Holland, (1973).

[21] W. Marciano and H. Pagels, Phys. Rept. 36, 137 (1978).

[22] M. L. Goldberger and S. B. Treiman, Phys. Rev. 111, 354 (1958).

[23] M. Gell-Mann, R. J. Oakes and B. Renner, Phys. Rev. 175, 2195 (1968).

TABLE I. Table of the flavor traces. $\operatorname{tr}\left\{\tau_{i 1} \tau_{i 2} \tau_{o 2}^{\dagger} \tau_{o 1}^{\dagger}\right\}$ and $\operatorname{tr}\left\{\tau_{i 1} \tau_{o 2}^{\dagger} \tau_{i 2} \tau_{o 1}^{\dagger}\right\}$ are examples of the direct and exchange cases.

\begin{tabular}{cccc}
\hline \hline$I m_{I}$ & $\tau_{i 1} \tau_{i 2}$ & direct & exchange \\
\hline 00 & $\frac{\bar{\sigma} \cdot \vec{\sigma}}{2 \sqrt{3}}$ & $\frac{3}{2}$ & $-\frac{1}{2}$ \\
11 & $\frac{\sigma_{1} \sigma_{2}-\sigma_{2} \sigma_{1}}{2 \sqrt{2}}$ & 1 & 0 \\
22 & $\sigma^{+} \sigma^{+}$ & 0 & 1 \\
\hline \hline
\end{tabular}

